\title{
Desenvolvimento de uma aplicação de realidade aumentada sobre conceitos de revolução e extrusão de polígonos
}

\author{
Christopher Shneider Cerqueira - UNIFEI - christophercerqueira@ gmail.com \\ Claudio Kirner - UNIFEI - ckirner@gmail.com
}

Resumo: As aplicações mais modernas de realidade aumentada estão tendendo a colocar objetos virtuais inteligentes no cenário, de forma a reagirem aos usuários e às condições do ambiente. Nesse sentido, este artigo trata de uma aplicação, voltada para o ensino matemático de conceitos de revolução e extrusão de polígonos, utilizando a ferramenta de autoria basAR. Isto cria um segmento de aplicações interativas de acesso a conteúdo, que possa ser utilizado como ferramenta educacional. $O$ artigo apresenta a construção da aplicação, os conceitos envolvidos e resultados de avaliação.

Palavras-chave: realidade aumentada; matemática; ensino.

Abstract: Nowadays, augmented reality application trends to insert smart virtual objects into the real scene in order to react to user and environment condition. In this way, this article shows an application to teach polygon extrusion and revolution math concepts, using the authoring tool bas AR, to create a set of interactive applications to access content that could be used as educational tool. This article presents the construction of the application, the concepts used and the evaluation results.

Keywords: Augmented reality; mathematics; education.

\section{Introdução}

O desenvolvimento de habilidades espaciais é um entendimento crítico, quando os estudantes passam a aprender os sólidos tridimensionais. Para desenvolver essa capacidade, geralmente são utilizadas maquetes de madeira e representações tridimensionais planas em livros (Gutierrez et al., 2009).

Uma possibilidade de melhorar a fixação da origem dos sólidos a partir de figuras em planos é utilizando realidade aumentada, cuja definição, segundo Kirner (2011a), é: "realidade aumentada é uma interface baseada na sobreposição de informações virtuais geradas por computador (envolvendo imagens estáticas e dinâmicas, sons espaciais e sensações hápticas) com o ambiente físico do usuário, percebida através de dispositivos tecnológicos e usando as interações naturais do usuário, no mundo físico".

As principais vantagens apontadas para a utilização de técnicas de RA para fins educacionais são as seguintes: (Cardoso, 2009).

a) Motivação de estudantes e usuários de forma geral, baseada na experiência vivida pelos mesmos.

b) Grande poderio de ilustrar características e processos, em relação a outras técnicas multimídias.

c) Permite visualização de detalhes e animações de objetos.

d) Suporte à visualização de objetos, que estão a grandes distâncias, como um planeta ou um satélite ou a pequenas distâncias como em estruturas atômicas;

e) Permite experimentos virtuais, na falta de recursos, ou para fins de educação virtual interativa;

f) Permite ao aluno refazer os experimentos fora da aula;

g) Devido à forma de interação, faz com que o aluno se torne ativo; 
h) Encoraja a criatividade, melhorando a experiência;

i) Provê igual oportunidade para estudantes de culturas diferentes;

j) Ensinar habilidades computacionais e de domínio de periféricos.

Um sistema que utiliza realidade aumentada como interface é a ferramenta Construct $3 D$. Esta é uma ferramenta de construção geométrica tridimensional, projetada especificamente para o estudo de matemática e geometria. A principal vantagem, neste caso, é que os estudantes podem visualizar e interagir com objetos, diretamente num espaço 3D, podendo compreender os conce itos de forma mais rápida que os métodos tradicionais (Construct3D, 2011).

Na Figura 1 são exibidos dois exemplos do uso da aplicação Construct3D.
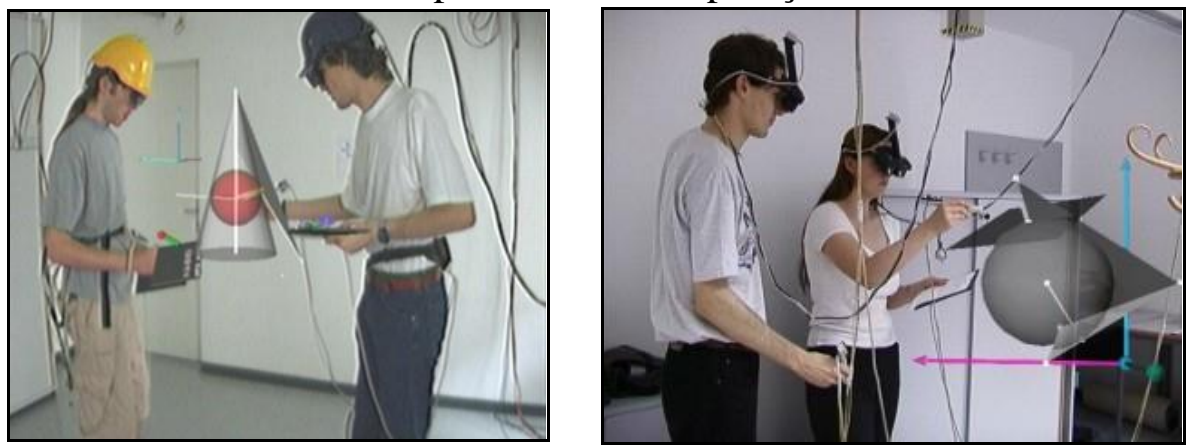

Figura 1 - Exemplos do uso do Construct3D

\section{A. basAR}

A ferramenta basAR (Behavioral Authoring System for Augmented Reality)(Cerqueira,2011a) é uma ferramenta de autoria de ambientes inteligentes de realidade aumentada que foi desenvolvida no âmbito do Projeto "Ambiente Temático Interativo com Realidade Aumentada", financiado pelo CNPq (Proc. 58842/2009-7) e FAPEMIG (Proc. APQ-03643-10). A Figura 2 mostra o marcador de base, com seus pontos de ação (esferas azuis) e o marcador de controle com o seu ponto de ação (usado para a interação do usuário com os pontos de ação do ambiente). Cada ponto virtual possui um raio de ação (esferas azuis transparentes), de forma que, quando um ponto entra no raio de ação de outro ponto, ocorre uma colisão. Na colisão entre o ponto do marcador de controle com os pontos da estrutura da base, ocorre algum comportamento como: ação, repulsão, depósito, mudança de modelo, etc.

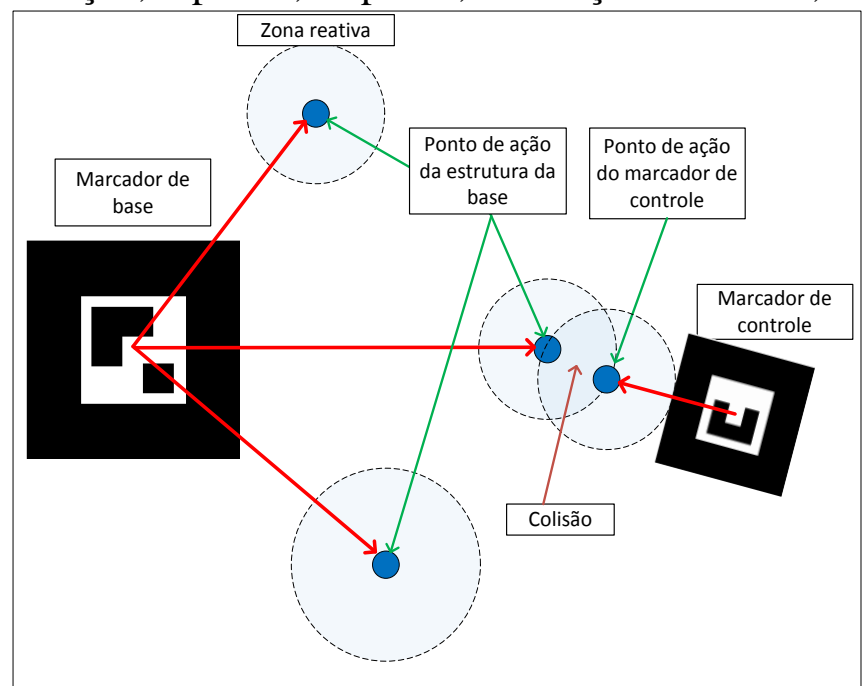

Figura 2 - Esquema do funcionamento do basAR. 
Alguns exemplos de utilização do bas AR incluem: sistemas de quebra cabeças, montagens virtuais ou jogos interativos, conforme ilustrações na Figura 3.
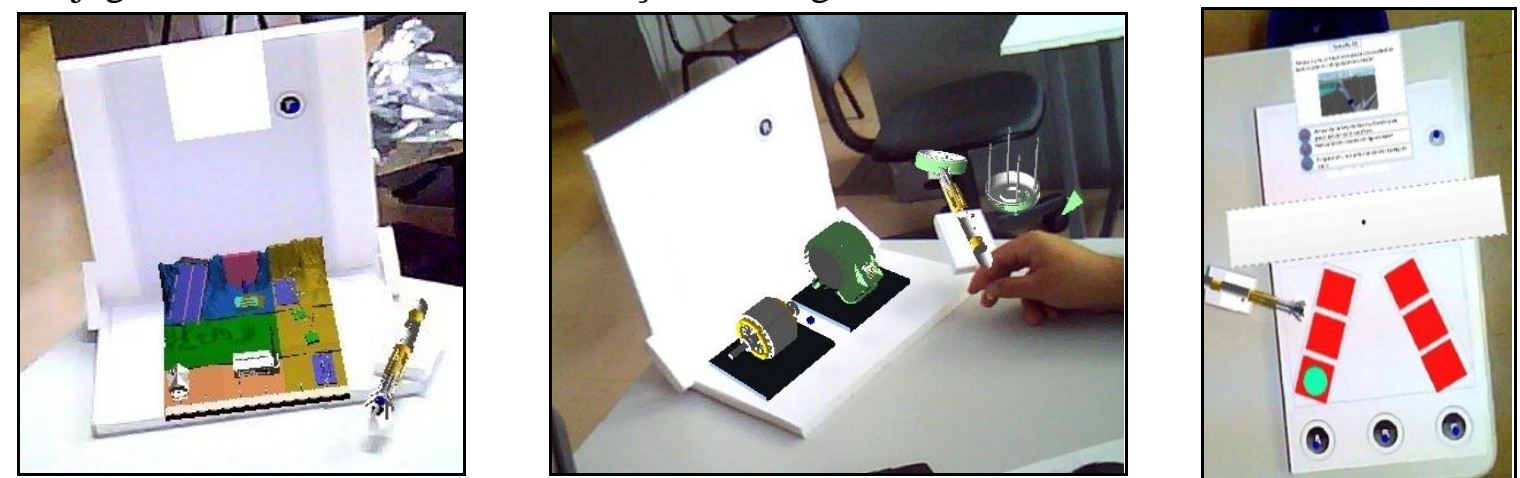

Figura 3 - Exemplos de utilização do basAR, da esquerda para a direita: quebra cabeças, montagem de motor e jogo interativo.

\section{Material e métodos}

\section{A. Concepção da aplicação}

A criação de uma aplicação de realidade aumentada com comportamento inicia-se com a definição da estrutura e funcionamento da aplicação. As descrição do funcionamento pode ser organizado num "fluxograma" de como a aplicação deve se comportar, de forma a construir uma lista de itens necessários para a criação da aplicação.

Para esta aplicação deseja-se construir um guia de conteúdo, onde, a partir da escolha de caminhos, o usuário che gue a um resultado. Este guia foi construído usando dois níveis de escolhas. No primeiro nível, escolhe-se um polígono em um conjunto de três figuras: círculo, quadrado e triângulo. Após selecionar a figura, escolhe-se uma ação em um conjunto de duas opções: extrusão e revolução. Após a escolha da ação, o sistema mos tra como a figura (polígono) é transformada na forma geométrica tridimensional (poliedro), decorrente da extrusão ou revolução da figura no plano.

A Figura 4 apresenta um esquema de como funciona o guia de conteúdo da escolha da figura até a exibição do poliedro obtido por extrusão ou revolução.

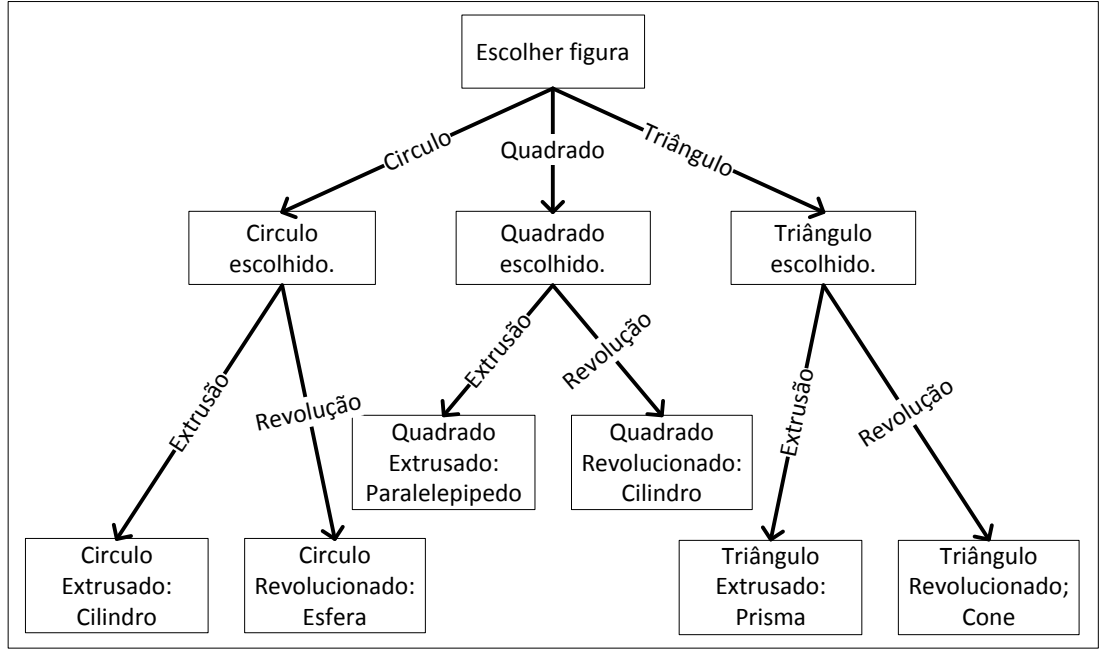

Figura 4 - Esquema do acesso ao conteúdo. 


\section{B. Criando infraestrutura e conteúdo}

A estrutura física da aplicação é a área de trabalho onde o usuário utilizará a aplicação. Para este artigo foi construída uma estrutura numa folha de papel, como na Figura 5a, com um marcador para relacionar a infraestrutura (correlação entre os itens virtuais com a estrutura física real). Na figura 5b estão os posicionamentos dos pontos de ação em relação ao marcador de infraestrutura. Nos pontos de ação são inseridos os comportamentos e a exibição do conteúdo virtual, como na Figura 5c.

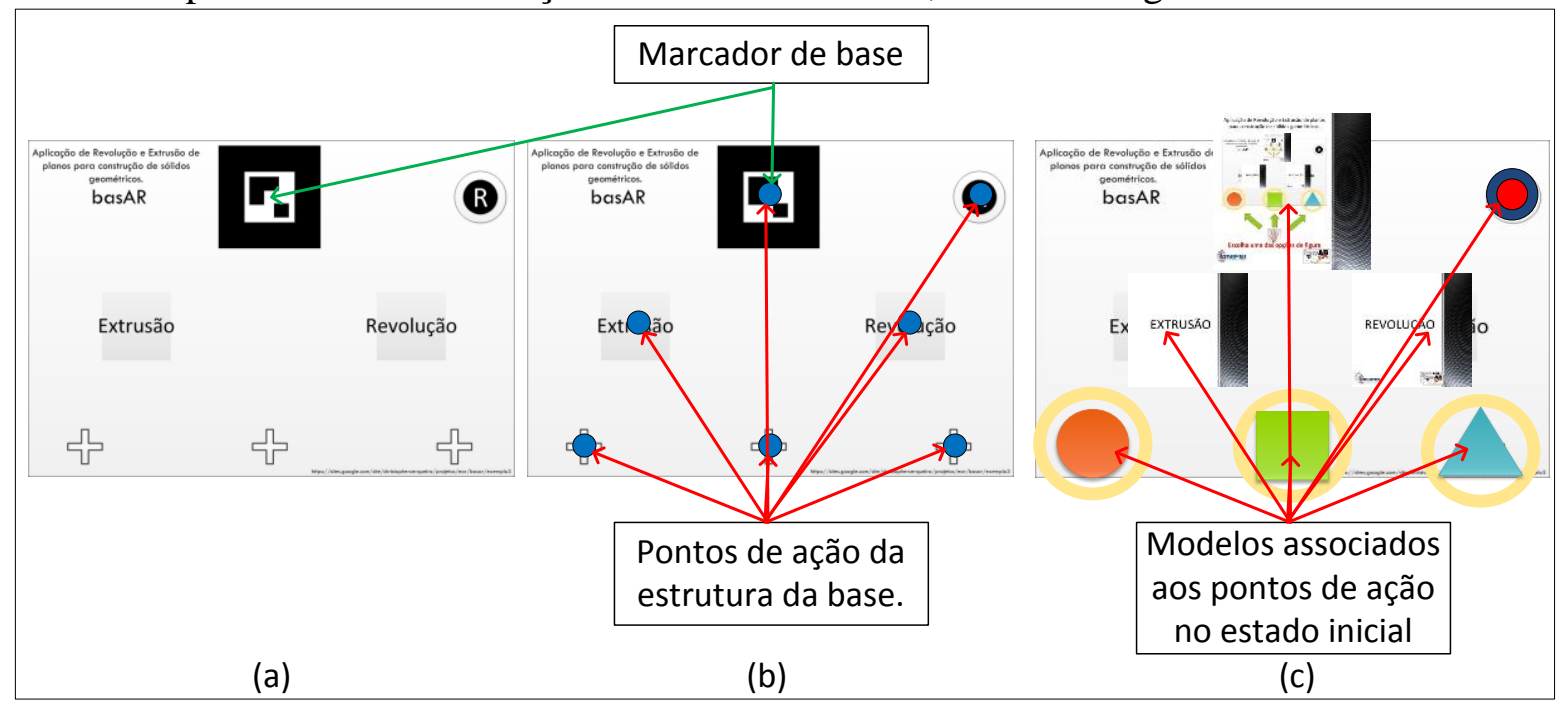

Figura 5 - (a) Estrutura física usada como área de trabalho; (b) Estrutura física com o posicionamento da estrutura virtual; (c) Modelos ativados da estrutura virtual.

Para interagir com os objetos da estrutura, é utilizado um artefato atuador. $\mathrm{O}$ atuador desta aplicação também utiliza um marcador. Nesta aplicação, foi construído um cubo feito de origami para ser distribuído, com instruções de montagem, conforme a Figura 6a. Quando montado, o cubo atuador fica semelhante ao exibido na Figura $6 b$.

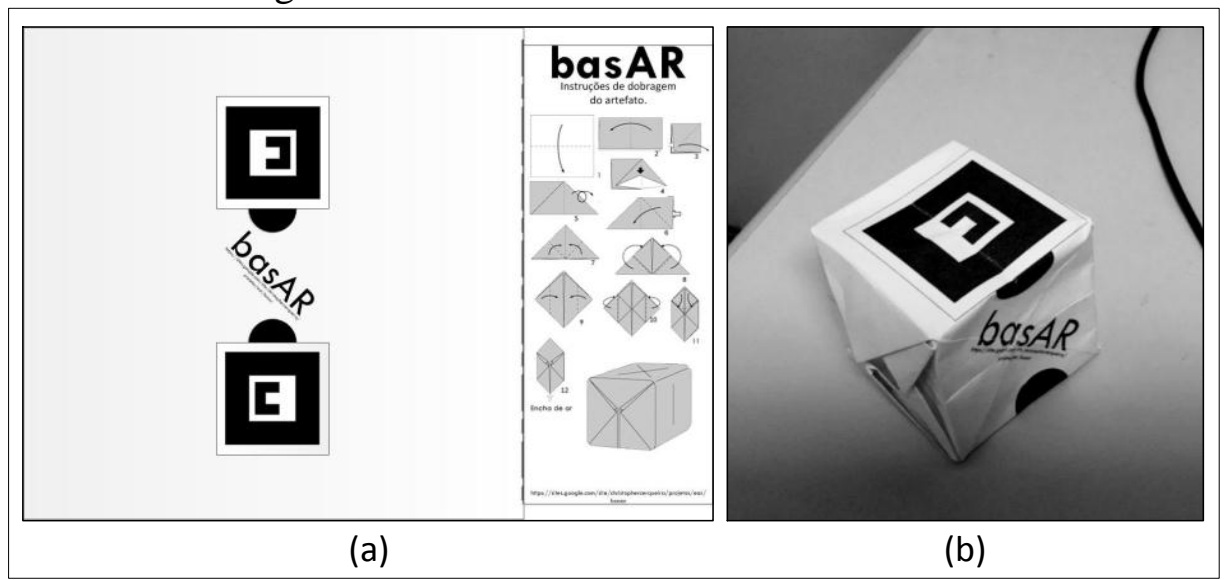

Figura 6 - Artefato atuador: (a) folha com instruções de montagem; (b) artefato montado.

Os conteúdos dos pontos de ação, correspondendo aos poliedros, foram criado utilizando o 3DStudioMax. O software Ms. Power Point foi utilizado para criar as texturas das placas explicativas. $\mathrm{Na}$ Figura 7a, é apresentada uma foto de como ficam posicionados os modelos 3D, identificando os modelos que não possuem textura e os que utilizam textura para passar alguma informação. Na Figura $7 b$, é exibido o modelo utilizado sobre artefato atuador e o ponto de ação (esfera azul). 


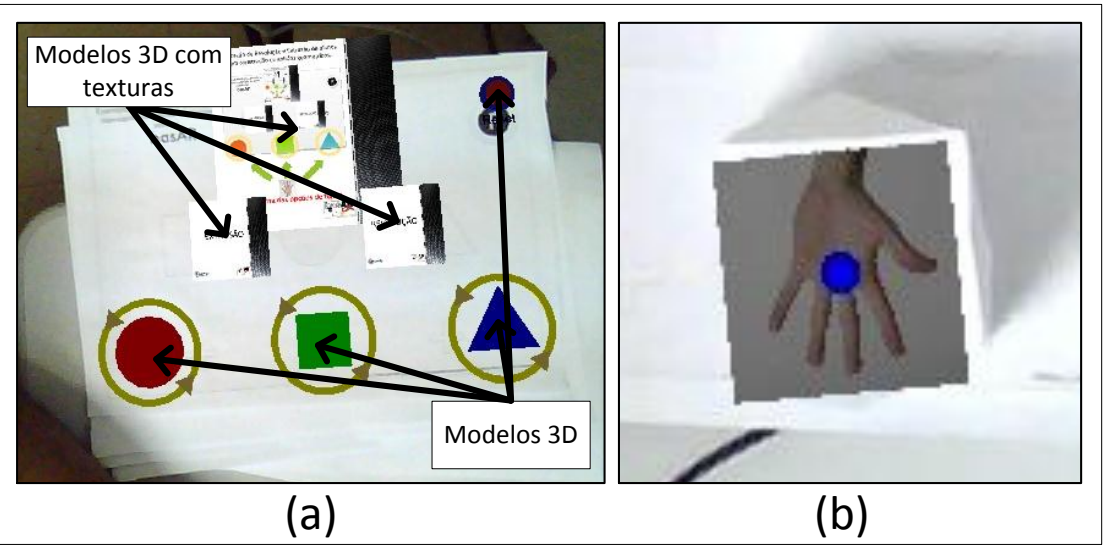

Figura 7 - (a) Modelos 3D com e sem textura; (b) Artefato atuador com modelo simbólico e ponto de colisão.

Foram construídos dois modelos para cada figura no plano: um mostrando uma seta circulando ao redor do modelo do plano, para dizer que está passível a habilitação, e quando selecionado mostra um elemento pulsante, utilizado para indicar que o objeto foi selecionado. Assim, foram construídos seis modelos: círculo girante, círculo pulsante, quadrado girante, quadrado pulsante, triângulo girante, triângulo pulsante

Para as placas informativas de extrusão e revolução, foram construídas: uma placa introdutória com o nome e três placas, informando como é a ação após a escolha do polígono. Assim, foram construídos oito modelos: introdução da extrusão, extrusão do círculo, extrusão do quadrado, extrusão do triângulo, introdução da revolução, revolução do círculo, revolução do quadrado e revolução do triângulo.

Para a placa grande de visualização, foram construídas placas informativas sobre o que fazer, e placas com o resultado da extrusão e da revolução. Assim, foram utilizados 10 modelos: introdução ao programa, auxílio para ação do círculo, auxílio para ação do quadrado, auxílio para ação do triângulo, resultado cilindro 1, resultado esfera, resultado paralelepípedo, resultado cilindro 2, resultado prisma, resultado cone.

\section{Definindo comportamento}

O comportamento foi programado seguindo a estrutura de comportamento do basAR (Cerqueira,2011b) e guiado pelo fluxograma da Figura 4, para criar o comportamento dos pontos de ação.

\section{Resultados e Discussão}

Este programa foi levado a teste para um grupo de alunos e professores da Universidade Federal de Itajubá, (UNIFEI), após uma palestra introdutória sobre a utilização do software basAR, sendo que o grupo testado possuía alto conhecimento sobre aplicações de realidade aumentada.

Após o teste deste aplicativo, foi passado um questionário com perguntas sobre interação, uso, aprendizagem, comportamentos, gráficos e funcionalidade. Utilizou-se a escala de Likert para avaliar o funcionamento da aplicação de matemática apresentada neste artigo.

\section{A. Interação}

Com relação à interação, os usuários foram questionados sobre a manipulação dos marcadores e as colisões, gerando o gráfico de avaliação da Figura 8: 


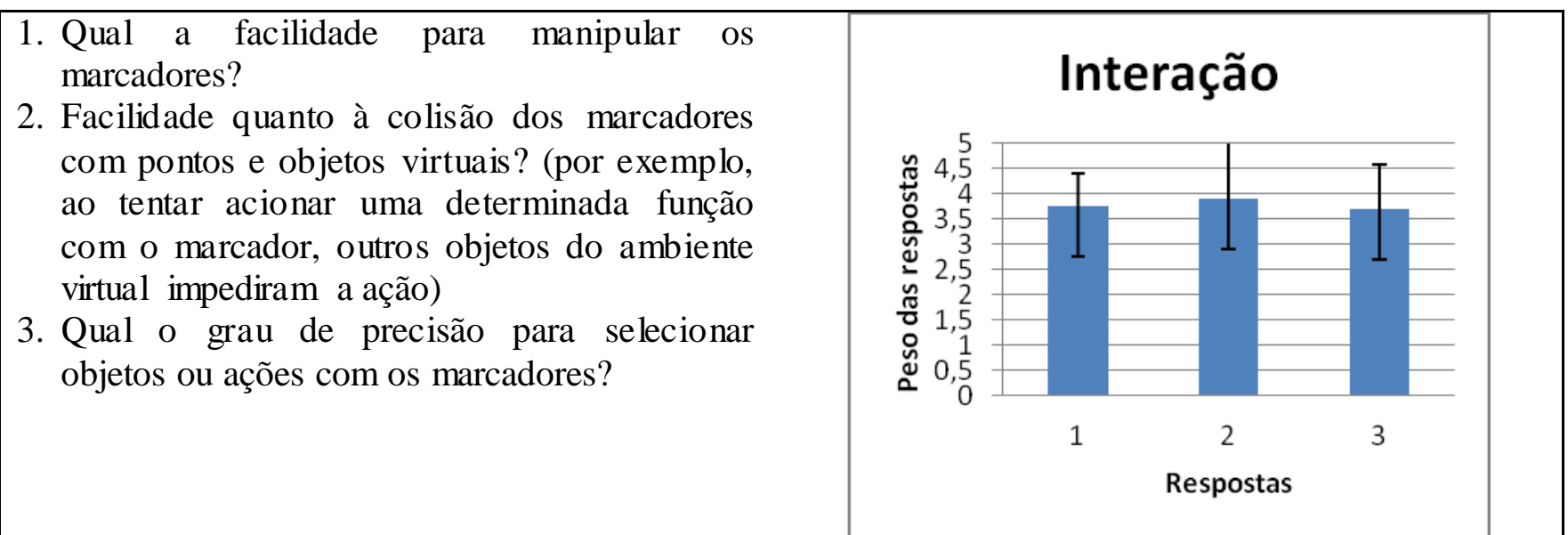

Figura 8 - Perguntas e gráfico das respostas sobre interação

Nas respostas livres, foi sugerido a utilização do marcador plano, ao invés do cubo, pois no grupo testado há o costume de utilizar-se um artefato atuador plano, devido a outros projetos. $\mathrm{O}$ artefato utilizado normalmente pelo grupo é vis to na Figura 9a e foi desenvolvido por Kirner (2011b), quando comparado ao cubo da Figura 9b.

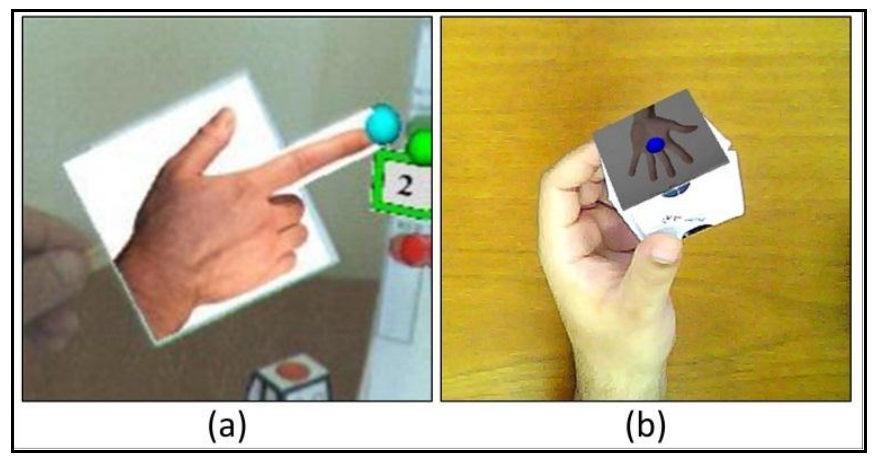

Figura 9 - Artefato atuador plano.

\section{B. Uso}

Com relação à facilidade de uso, os usuários foram questionados sobre a usabilidade do programa, gerando o gráfico de avaliação da Figura 10:

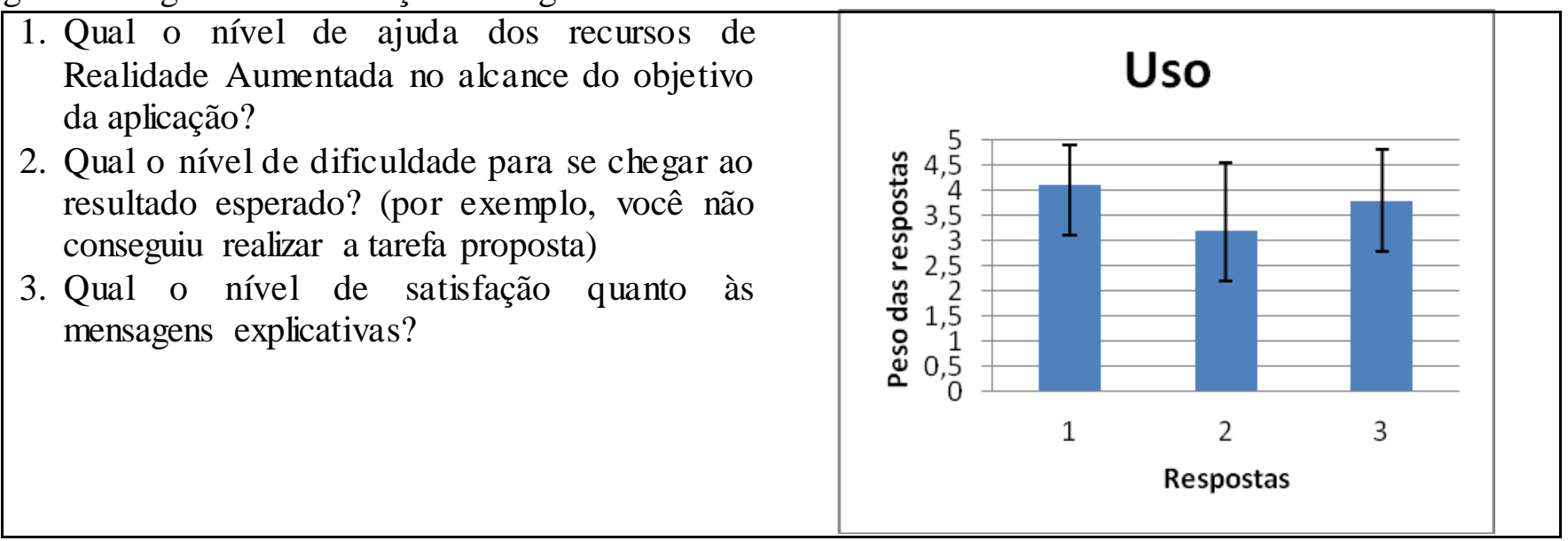

Figura 10 - Perguntas e gráfico das respostas sobre usabilidade. 
Nas respostas livres, foi sugerido o aumento das fontes dos textos explicativos, orientando melhor a condução da atividade.

\section{Aprendizagem}

Com relação à facilidade de aprendizagem, foi perguntado a respeito da facilidade de utilização da aplicação, gerando o gráfico de avaliação da Figura 11:

1. Qual o nível de facilidade quanto ao aprendizado do funcionamento e da utilização da aplicação?

2. Quão fácil é a memorização das regras de utilização da aplicação?

3. A aplicação se mostra interativa? (você é envolvido pela aplicação)

4. A aplicação é atraente? (ela chama atenção mesmo antes de se iniciar a aplicação)

\section{Aprendizagem}

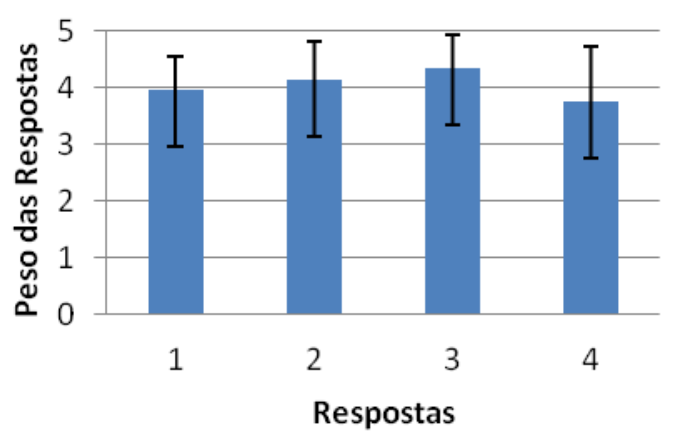

Figura 11 - Perguntas e gráfico das respostas sobre aprendizagem.

\section{Comportamento}

A respeito do comportamento, os usuários manifestaram-se sobre as características do comportamento da aplicação, gerando o gráfico da Figura 12:

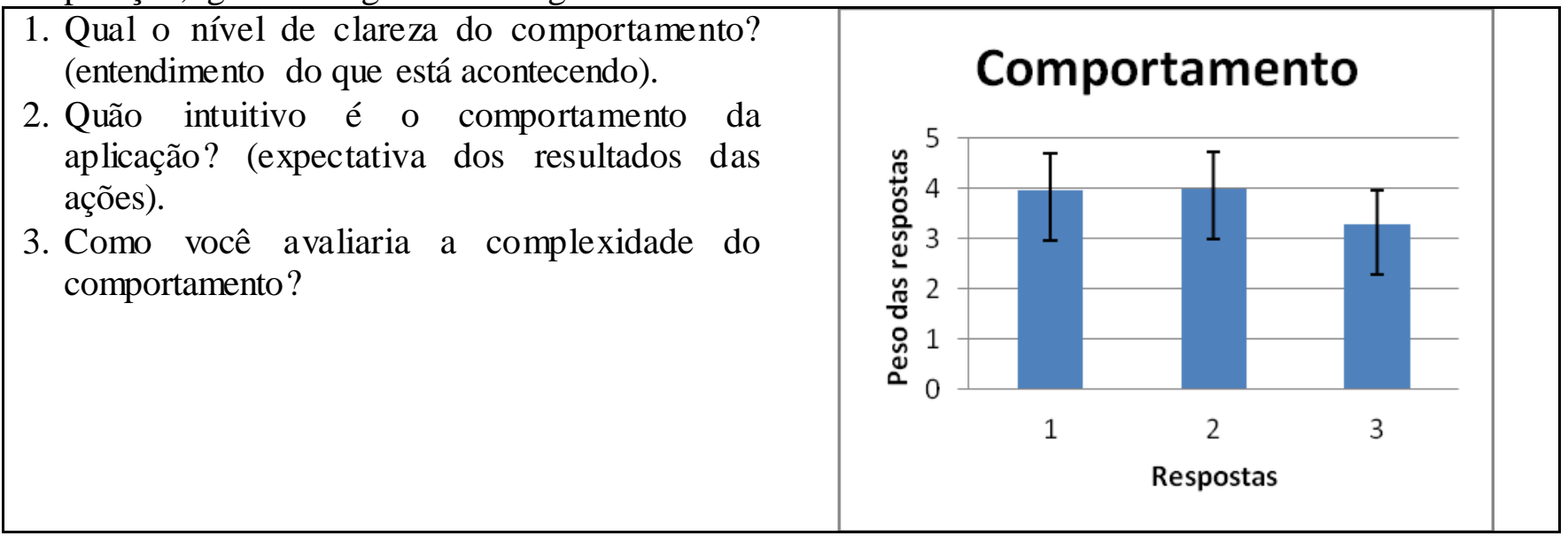

Figura 12 - Perguntas e gráfico das respostas sobre comportamento.

\section{E. Gráficos}

Sobre os gráficos, os usuários manifestaram-se quanto à qualidade visual dos elementos, gerando o gráfico de avaliação da Figura 13: 
1. Qual o nível de qualidade dos gráficos $\mathrm{e}$ imagens usados? (cores, contraste, aspecto visual em si)

2. Qual o nível de capacidade da aplicação de manter a sua concentração e atenção?

3. Qual o nível de clareza dos textos apresentados? (tipo de fonte, tamanho, cor, contraste, brilho, intensidade)

4. Qual o nível de clareza dos textos, quanto à facilidade de visualização?

5. Qual o nível de qualidade dos objetos 3D?

6. Qual o nível de satisfação da integração dos recursos físicos e visuais? (por exemplo, houve alguma dificuldade com a manipulação dos marcadores)

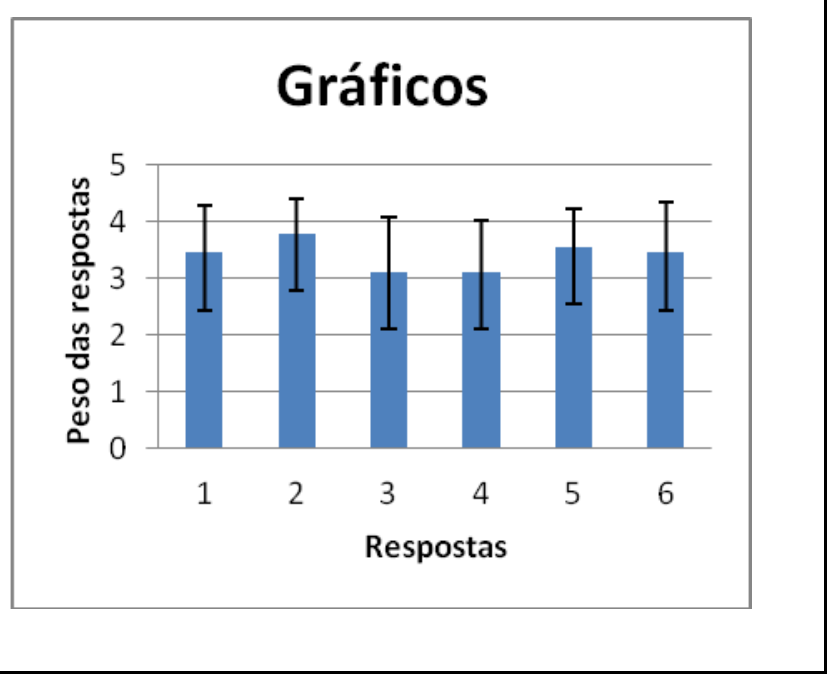

Figura 13 - Perguntas e gráfico das respostas sobre gráficos.

\section{F. Funcionalidade}

Sobre a funcionalidade, foi perguntado se a aplicação é pertinente ao contexto apresentado, gerando o gráfico de avaliação da Figura 14:

\begin{tabular}{|l|l|l|l|l|}
\hline 1. Qual o nível de entendimento do assunto & \multicolumn{3}{c|}{ Funcionalidade } \\
abordado? \\
2. Qual o nível de fixação do conceito de \\
revolução e extrusão? \\
3. Qual o nível de representação dos modelos \\
com as funções propostas? \\
\end{tabular}

Figura 14 - Perguntas e gráfico das respostas sobre funcionalidade.

O resumo das avaliações consta na Figura 15, permitindo fazer-se uma comparação sobre o desempenho dos vários itens avaliados. 


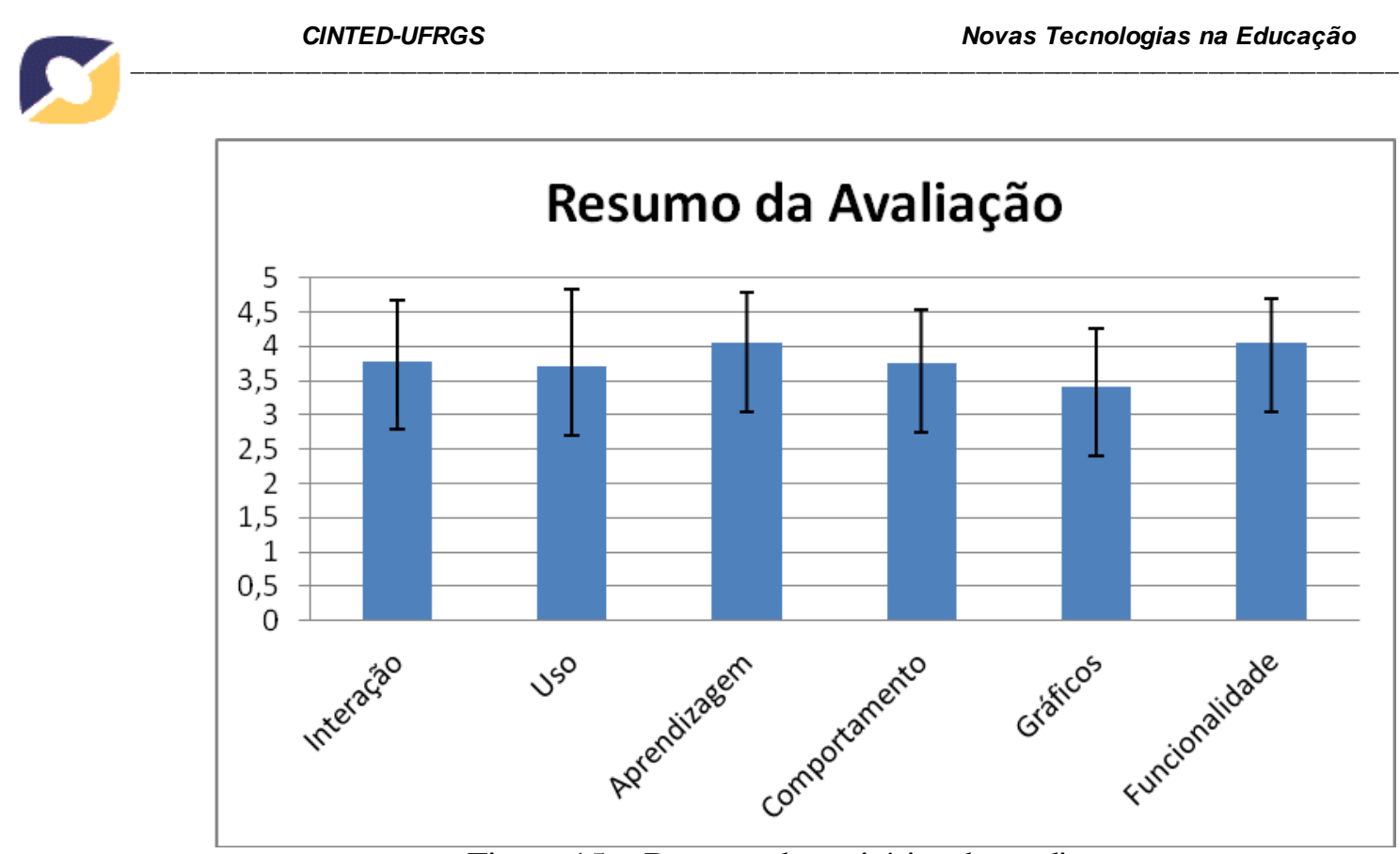

Figura 15 - Resumo dos critérios de avaliação.

\section{Conclusões}

A realidade aumentada é uma ferramenta poderosa para tornar as atividades educacionais mais criativas e motivar o ensino e a aprendizagem.

A ferramenta basAR permite criar ambientes de realidade aumentada de forma facilitada, sem que haja a necessidade de programar numa linguagem de programação. Os desenvolvedores da ferramenta, bem como alunos envolvidos em projetos correlatos, estão desenvolvendo templates para facilitar ainda mais a utilização da ferramenta, de forma que a prototipagem da aplicação seja mais rápida e segura. Templates, neste contexto, são aplicações genéricas que executam atividades específicas, de forma que, com alterações simples na descrição dos pontos, modelos e sons, pode-se gerar aplicativos específicos.

A aplicação de matemática (geometria), usando extrusão e revolução, é a primeira de uma série de aplicativos que serão disponibilizados para a utilização. Essas aplicações envolvem a manipulação de conteúdos, utilizando recursos de interação da interface de realidade aumentada que o basAR disponibiliza.

A avaliação da aplicação, resumida na Figura 15, indicou algumas características não observadas, como a disponibilização de mais do que um artefato de atuação, incluindo a versão apresentada do cubo e uma versão plana, como na Figura 9, devido ao grupo envolvido já ter experiência com outro artefato. Porém, a utilização do cubo é uma possibilidade de baixo custo, pois depende apenas da impressão do papel, com as instruções de montagem.

Outra informação, obtida dos testes, foi a necessidade de aumentar as fontes das figuras, pois com a área de trabalho mais distante, as instruções ficam mais difíceis de serem vistas.

A aplicação está disponível para download em:

- http://www.ckirner.com/basar

- https://sites.google.com/site/christophercerqueira/projetos/ear/basar. 


\section{Referências bibliográficas}

MARTÍN-GUTIÉRREZ, J.; SAORÍN, J. L.; CONTERO, M.; ALCAÑIZ, M.; PÉREZ-LÓPEZ, D. C.; ORTEGA, M. Design and validation of an augmented book for spatial abilities development in engineering students. Computer \& Graphics. Jan. 2009. Disponível em: < www.elsevier.com/locate/cag> Acesso em: 12 out. 2011.

KIRNER, C. Prototipagem Rápida de Aplicações Interativas de Realidade Aumentada. In: Tendências e Técnicas em Realidade Virtual e Aumentada. Porto Alegre - RS. SBC, 2011. p. 29-54.

CARDOSO, A.; LAMONIER JR, E. Aplicações de RV e RA na Educação e Treinamento. In: Aplicações de Realidade Virtual e Aumentada. Porto Alegre - RS. SBC, 2011. p. 53-68.

CONSTRUCT3D. Interactive Media Systems Group. Desenvolvido pelo Institute for Software Technology and Interactive Systems da Vienna University of Technology. Disponível em: <http//www.ims.tuwien.ac.at/research/construct3d/>. Acesso em: 12 out. 2011.

CERQUEIRA, C. S.; KIRNER C. basAR: Ferramenta de Autoria de Realidade Aumentada com Comportamento. Anais do VIII Workshop de Realidade Virtual e Aumentada. Uberaba, MG, 2 p, 2011. Disponível em: < https://sites.google.com/site/christophercerqueira/projetos/ear> Acesso em: 12 out. 2011.

CERQUEIRA, C. S.; KIRNER C. Programando Comportamento de Pontos de Ação em Aplicações de Realidade Aumentada. Anais do VIII Workshop de Realidade Virtual e Aumentada. Uberaba, MG, 2p, 2011. Disponível em: < https://sites.google.com/site/christophercerqueira/projetos/ear> Acesso em: 12 out. 2011.

KIRNER, C.; KIRNER, T. G. Educational Spatial Game using an Augmented Reality Authoring Tool. In: International Journal of Computer Information Systems and Industrial Management Applications. MIR Labs. ISSN 2150-7988. Volume 3. 2011.pp. 602-611 